\title{
Real-Time Estimation of a Ship's Attitude
}

\author{
Sebastian Küchler, Christoph Pregizer, Johannes Karl Eberharter, Klaus Schneider, and Oliver Sawodny
}

\begin{abstract}
During subsea lifting operations in harsh sea conditions, the involved crane system is subjected to extensive dynamic forces due to vertical vessel motion. Thus, active heave compensation systems can be used to compensate for vertical vessel motion and to reduce forces acting on the crane structure. Furthermore, such systems allow an exact positioning of the load on the seabed. However, active heave compensation systems always require knowledge about the vertical position of the crane depending on the ship's heave, roll, and pitch motion. Hence, an attitude estimation method for ships during subsea lifting operations is proposed. To estimate the roll and pitch motion of a vessel with high accuracy, rotation rate sensors are fused with accelerometers using an Extended Kalman Filter. Since an exact knowledge of the yaw motion is not required to determine the crane's vertical motion, the yaw angle is stabilized around zero with an additional virtual sensor signal. The attitude estimation algorithm is evaluated with simulation and measurement results from an experimental setup.
\end{abstract}

\section{INTRODUCTION}

There is an increasing demand for offshore installations, such as underwater conveying systems for oil and gas fields or wind parks in the near future. Offshore oil and gas fields will be developed to a large extent with all processing equipment on the seabed. Thus, high operability on the underwater construction is required.

On the other hand, dealing with such installations puts new challenges on the equipment for the offshore industry, because waves, wind, and ocean currents easily cause the vessel to move away both horizontally and vertically. Especially, the vessel's vertical motion has a significant effect on the involved crane system shown in Fig. 1 during subsea lifting operations. Thus, it is important to keep the load motion unaffected by the wave induced vessel motion even under harsh sea conditions. For decoupling the vertical load motion from the vertical vessel motion, passive or active heave compensation systems can be used. All active systems have in common that they require exact knowledge of the vertical crane tip's position where the load is attached to, as described in the literature [1], [2], [3]. However, the vertical motion of the crane tip is not only affected by the vessel's heave motion, but also by the vessel's roll and pitch motion. Hence, active heave compensation systems require the actual heave, roll, and pitch motion of a vessel.

All six degrees of freedom of a vessel or ship can be obtained from an inertial measurement unit (IMU). Such a standalone

\footnotetext{
S. Küchler, C. Pregizer, and O. Sawodny are with the Institute for System Dynamics, University of Stuttgart, P.O. Box 801140, D-70511 Stuttgart, Germany sebastian.kuechler@isys.uni-stuttgart.de

J. K. Eberharter and K. Schneider are with Liebherr Werk Nenzing GmbH, P.O. Box 10, A-6710 Nenzing, Austria
}

motion sensor has three accelerometers for measuring surge, sway, and heave and three rotation rate sensors for roll, pitch, and yaw. Obviously, the accelerometer signals have to be integrated twice and the rotation rate signals once to obtain the relative position and attitude of a vessel. To reduce typical errors like sensor noise, bias, and misalignment of the rotation rate sensors and accelerometers, different approaches for signal conditioning can be found in the literature. For example, Godhavn [4] proposes integrating filters to obtain the relative position of a vessel. The filter coefficients are chosen in accordance to the actual sea spectrum. Other approaches for attitude estimation of a body fuse different sensor signals to compensate different errors of each sensor. A widely-used approach is to aid the IMU signals with GPS measurements to eliminate the resulting drift terms of the estimated position and attitude as described by Godhavn [5] or Fossen and Perez [6]. However, the proposed method requires additional GPS signals resulting in higher costs.

Another possibility for attitude estimation is to fuse the signals of the gyro rate sensors with the measured accelerations of the IMU to estimate the errors of the rotation rate signals. Kim and Golnaraghi [7] propose such a method. They model the rotation rates as first order systems and formulate an Extended Kalman Filter (EKF) estimating the attitude of a rigid body. Metni et al. [8] fuse signals of three gyroscopes, three accelerometers, and three magnetometers in two complementary filters. The derived filters are used to estimate the attitude of an unmanned aerial vehicle. The first filter fuses the gyroscopes with the accelerometers to estimate the roll and pitch angles, while the second filter is used to aid the gyroscopes with the magnetometers to estimate the yaw angle. The additional usage of magnetometers results in a better estimation of the yaw angle. Data fusion of three gyroscopes, two inclinometers, and a compass via an EKF is presented by Setoodeh et al. [9]. The paper derives an indirect error state model basing on attitude errors and bias of the gyroscopes. The resulting EKF is realized in a way that it separates the model of the bias and the error dynamics resulting in two subsystems. However, all these methods have problems estimating the correct roll and pitch angles in situations when the IMU is accelerated, since they use a model of the earth's gravitational vector, which is only valid for an IMU that is not accelerated, to stabilize the roll and pitch motion.

This paper presents a method for estimating the attitude of a ship or vessel during subsea lifting operations. The scheme utilizes a low-cost IMU (ADIS 16365) from Analog Devices as a standalone motion sensor without aiding external sensor sources like a GPS receiver or a compass. The IMU has 


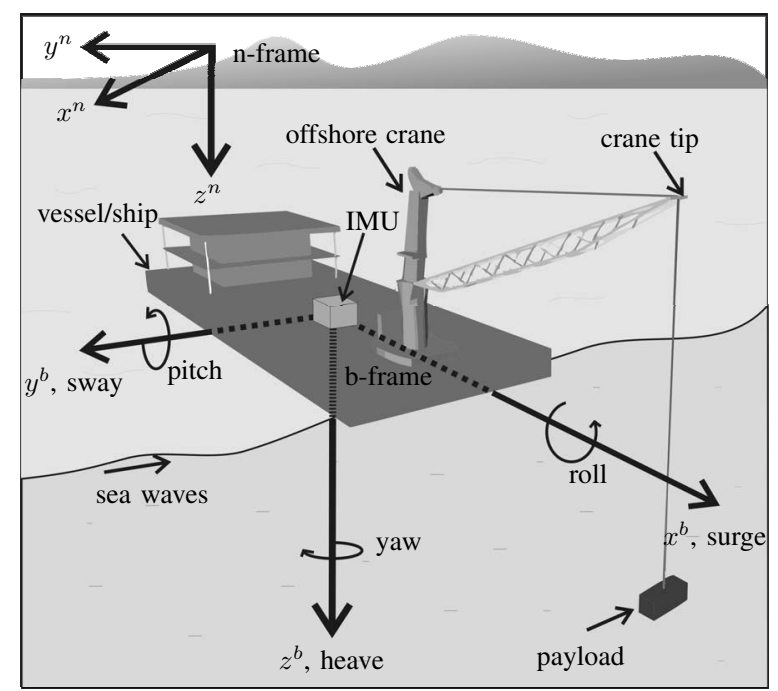

Fig. 1. Vessel/Ship with offshore crane.

three accelerometers and three rotation rate sensors. To avoid dependency on the knowledge about a certain magnetic field, the algorithm does not use additional magnetometers. Special attention is given to the roll and pitch motion, as these influence the vertical motion of a crane during subsea lifting operations as depicted in Fig. 1. Exact knowledge of the yaw motion is not required for active heave compensation systems as it does not result in a vertical motion of the crane tip that must be compensated. To estimate the roll and pitch motion, the rotation rate signals of the gyroscopes are fused with the accelerometers using an EKF. To guarantee good performance also in situations when the IMU is accelerated, the covariance matrices of the EKF are adapted online using the absolute values of the measured accelerations and rotation rates. Furthermore, it is shown that a virtual sensor for the yaw angle can be used to aid the estimated yaw angle and to stabilize it around zero to avoid an unbounded drift of this angle resulting in numerical problems.

\section{SENSOR MODELING}

In the following a dynamic model for an orientation sensor is derived. The sensor used in this work is a standard low-cost strapdown IMU (ADIS 16365) from Analog Devices. The IMU consists of three rate gyros and three accelerometers in orthogonal triads. The objective of the model is to design an observer estimating the pitch and roll angles of a ship or vessel during subsea lifting operations without requiring any ship specific parameters. Thus it is desired that the model only consists of sensor specific parameters or dynamics.

As depicted in Fig. 1, there are two main frames used in the following. The navigation frame, denoted by $n$, is defined as a North-East-Down frame (NED-frame) with the vertical axis directing toward the center of the earth (down). Because the ship stays at a fixed position during subsea lifting operations, the $n$-frame is fixed to the earth. Furthermore, the effects due to the rotation of the earth are considered as negligible, since these effects are in the range of the sensor noise; hence, the $n$-frame is considered as the inertial frame. The second frame is the body frame, denoted by $b$, and attached to the IMU. The orthogonal axes are aligned with the sensor axes.

The rotation model of the sensor is derived using quaternions rather than direction cosines and Euler angles, as the quaternion representation does not have a problem with singularities, handles normalization better, and does not require bulky trigonometric calculations of Euler angles. Following [10], the propagation of the quaternion $\mathbf{q}_{n}^{b}=\left[\begin{array}{llll}q_{0} & q_{1} & q_{2} & q_{3}\end{array}\right]^{T}$, indicating the transformation from the $n$ - to the $b$-frame, can be expressed using the rotation rates $\omega_{n b}^{b}=\left[\begin{array}{lll}\omega_{x} & \omega_{y} & \omega_{z}\end{array}\right]^{T}$ (rotation rate of the $b$-frame relative to the $n$-frame expressed in the $b$-frame) and written as

$$
\begin{aligned}
& \dot{q}_{0}=-\frac{1}{2}\left(q_{1} \omega_{x}+q_{2} \omega_{y}+q_{3} \omega_{z}\right), \\
& \dot{q}_{1}=\frac{1}{2}\left(q_{0} \omega_{x}-q_{3} \omega_{y}+q_{2} \omega_{z}\right), \\
& \dot{q}_{2}=\frac{1}{2}\left(q_{3} \omega_{x}+q_{0} \omega_{y}-q_{1} \omega_{z}\right), \\
& \dot{q}_{3}=-\frac{1}{2}\left(q_{2} \omega_{x}-q_{1} \omega_{y}-q_{0} \omega_{z}\right) .
\end{aligned}
$$

Because every gyroscope measures an additive offset and sensor noise to the rotation rate itself, the measured rates of the IMU $\boldsymbol{\omega}_{\mathrm{imu}}^{b}$ are not the same as the actual rates of the body $\boldsymbol{\omega}_{n b}^{b}$. Thus, each measured rate of the IMU is modeled as the sum of the actual body rate, an offset term, and random sensor noise. The resulting rotation rates measured through the IMU are given by

$$
\boldsymbol{\omega}_{\mathrm{imu}}^{b}=\boldsymbol{\omega}_{n b}^{b}+\boldsymbol{\rho}+\boldsymbol{\xi}_{\boldsymbol{\omega}},
$$

where $\boldsymbol{\rho}=\left[\begin{array}{lll}\rho_{x} & \rho_{y} & \rho_{z}\end{array}\right]^{T}$ and $\boldsymbol{\xi}_{\boldsymbol{\omega}}=\left[\begin{array}{lll}\xi_{\omega_{x}} & \xi_{\omega_{y}} & \xi_{\omega_{z}}\end{array}\right]^{T}$ are the offset term and sensor noise of the gyroscopes.

The dynamic behavior of the actual body rates $\boldsymbol{\omega}_{n b}^{b}$ itself is assumed as a first order system with additive white noise as unknown input as it is proposed by Kim and Golnaraghi [7]. Thus the ODE for each body rate may be expressed as

$$
\begin{aligned}
& \dot{\omega}_{x}=\frac{1}{\tau_{\omega}} \omega_{x}+\zeta_{\omega_{x}}, \\
& \dot{\omega}_{y}=\frac{1}{\tau_{\omega}} \omega_{y}+\zeta_{\omega_{y}}, \\
& \dot{\omega}_{z}=\frac{1}{\tau_{\omega}} \omega_{z}+\zeta_{\omega_{z}}
\end{aligned}
$$

with the time constant $\tau_{\omega}>0$ and the additive white noise $\boldsymbol{\zeta}_{\boldsymbol{\omega}}$. The time constant $\tau_{\omega}$ reflects the expected dynamics of the rotation rates of the body. In the following, a very slow dynamic is assumed for the offset term in (2). Hence, each gyro offset is modeled as a random walk process with additive white noise, yielding the following expression:

$$
\dot{\rho}=\zeta_{\rho},
$$

where $\zeta_{\rho}=\left[\zeta_{\rho_{x}} \zeta_{\rho_{y}} \zeta_{\rho_{z}}\right]^{T}$ denotes the offsets' white noise. It is clear that a direct integration of the measured rates $\boldsymbol{\omega}_{\text {imu }}^{b}$ using (1) would result in a drift of the quaternion $\mathbf{q}_{n}^{b}$, due to the gyro offsets. Therefore, the rate sensors of the IMU have to be aided by an additional sensor for a drift-free integration of the quaternion. Since it is desired that the IMU acts as a standalone sensor for the vessel's attitude estimation, the accelerometers of the IMU are used to compensate the gyro offsets. The basic principle of aiding rotation rate sensors with accelerometers is the fact that the 
accelerometers measure only the earth's gravitation in the $b$ frame during phases when the IMU is not accelerated by other forces. Furthermore, the earth's gravitational vector measured by the IMU always directs toward the negative $z$-axis in the $n$-frame. Thus the measured accelerations in the $b$-frame during phases when the gravitational vector only acts on the IMU may be written as

$$
\mathbf{a}_{\mathrm{imu}}^{b}=\mathbf{C}_{n}^{b}\left[\begin{array}{lll}
0 & 0 & -\tilde{\mathbf{g}}
\end{array}\right]^{T}+\boldsymbol{\xi}_{\boldsymbol{a}},
$$

where $\tilde{\mathbf{g}}=\|\mathbf{g}\|$ denotes the absolute value of the earth's gravitation and $\boldsymbol{\xi}_{\boldsymbol{a}}=\left[\begin{array}{lll}\xi_{a_{x}} & \xi_{a_{y}} & \xi_{a_{z}}\end{array}\right]^{T}$ is the additive sensor noise of the accelerometers. The transformation matrix $\mathbf{C}_{n}^{b}$ from the $n$ - to the $b$-frame using the quaternion representation is given by (see [10])

$$
\begin{aligned}
\mathbf{C}_{n}^{b}= & {\left[\begin{array}{cc}
q_{0}^{2}+q_{1}^{2}-q_{2}^{2}-q_{3}^{2} & 2 q_{1} q_{2}+2 q_{0} q_{3} \\
2 q_{1} q_{2}-2 q_{0} q_{3} & q_{0}^{2}-q_{1}^{2}+q_{2}^{2}-q_{3}^{2} \\
2 q_{1} q_{3}+2 q_{0} q_{2} & 2 q_{2} q_{3}+2 q_{0} q_{1} \\
& 2 q_{1} q_{3}-2 q_{0} q_{2} \\
& 2 q_{2} q_{3}-2 q_{0} q_{1} \\
& q_{0}^{2}-q_{1}^{2}-q_{2}^{2}+q_{3}^{2}
\end{array}\right] . }
\end{aligned}
$$

Hence, using (5) and (6), the measured accelerations of the IMU due to the gravitational vector may be written as

$$
\begin{aligned}
& a_{x, \text { imu }}^{b}=\left(2 q_{0} q_{2}-2 q_{1} q_{3}\right) g+\xi_{a_{x}}, \\
& a_{y, \text { imu }}^{b}=-\left(2 q_{0} q_{1}+2 q_{2} q_{3}\right) g+\xi_{a_{y}}, \\
& a_{z, \text { imu }}^{b}=\left(-q_{0}^{2}+q_{1}^{2}+q_{2}^{2}-q_{3}^{2}\right) g+\xi_{a_{z}} .
\end{aligned}
$$

Due to a higher clearness of Euler angles compared to a quaternion representation, Euler angles are used in the following to depict the results and explain certain effects of the proposed algorithm. In this research, the order of successive rotations expressing the transformation from the $n$ - to the $b$-frame with Euler angles is defined as yaw-pitchroll [10] yielding the following relationship:

$$
\begin{aligned}
\tan (\Psi) & =\frac{2\left(q_{1} q_{2}+q_{0} q_{3}\right)}{q_{0}^{2}+q_{1}^{2}-q_{2}^{2}-q_{3}^{2}}, \\
\sin (\Theta) & =-2\left(q_{1} q_{3}-q_{0} q_{2}\right), \\
\tan (\Phi) & =\frac{2\left(q_{2} q_{3}+q_{0} q_{1}\right)}{q_{0}^{2}-q_{1}^{2}-q_{2}^{2}+q_{3}^{2}} .
\end{aligned}
$$

Here, $\Psi, \Theta$, and $\Phi$ denote the yaw, pitch, and roll angles around the $z, y$, and $x$ axis of the respective coordinate systems.

\section{OBSERVER DESIGN}

The derived dynamical model of the gyroscope sensors can be used to design an observer estimating the attitude of a ship. Obviously, the objective of designing an observer that estimates the ship's roll and pitch angle without any drift can be achieved using the properties of the earth's gravitational vector (7). However, a drift-less estimation of the yaw angle is not possible using (7), since a yaw motion does almost not affect the direction of the earth's gravitational vector in the $b$-frame. To avoid an unbounded drift of the yaw angle that results in numerical problems, an additional relation is required. Since a ship does not perform great yaw motion during subsea lifting operations, it can be assumed that the relative yaw angle oscillates around zero. Hence, a virtual sensor signal is used in the following to reflect the property that the yaw angle oscillates around zero. The output equation of the virtual sensor is given by (8) with $\Psi=0$.

To design an observer, the derived sensor model and the relationship for the virtual sensor have to be transformed to a state space model. Defining the state vector as $\mathbf{x}=$ $\left[\begin{array}{lll}\mathbf{q}_{n}^{b} & \boldsymbol{\omega}_{n b}^{b} & \boldsymbol{\rho}\end{array}\right]^{T}$ and neglecting the IMU's sensor noise in (2) and (7) as well as the process noise in (3) and (4), equations (1) - (4), (7), and (10) can be transformed to state space form given by

$$
\begin{aligned}
& \dot{\mathbf{x}}=\mathbf{f}(\mathbf{x})=\left[\begin{array}{c}
-\frac{1}{2}\left(x_{2} x_{5}+x_{3} x_{6}+x_{4} x_{7}\right) \\
\frac{1}{2}\left(x_{1} x_{5}-x_{4} x_{6}+x_{3} x_{7}\right) \\
\frac{1}{2}\left(x_{4} x_{5}+x_{1} x_{6}-x_{2} x_{7}\right) \\
-\frac{1}{2}\left(x_{3} x_{5}-x_{2} x_{6}-x_{1} x_{7}\right) \\
\frac{1}{\tau_{\omega}} x_{5} \\
\frac{1}{\tau_{\omega}} x_{6} \\
\frac{1}{\tau_{\omega}} x_{7} \\
0 \\
0 \\
0
\end{array}\right] \\
& \mathbf{y}=\mathbf{h}(\mathbf{x})=\left[\begin{array}{c}
\left(2 x_{1} x_{3}-2 x_{2} x_{4}\right) g \\
-\left(2 x_{1} x_{2}+2 x_{3} x_{4}\right) g \\
\left(-x_{1}^{2}+x_{2}^{2}+x_{3}^{2}-x_{4}^{2}\right) g \\
x_{5}+x_{8} \\
x_{6}+x_{9} \\
x_{7}+x_{1} 0 \\
\frac{2\left(x_{2} x_{3}+x_{1} x_{4}\right)}{x_{1}^{2}+x_{2}^{2}-x_{3}^{2}-x_{4}^{2}}
\end{array}\right] \text {. }
\end{aligned}
$$

Note that the last row of the system's output vector $y_{7}$ stands for the virtual sensor signal with the corresponding measurement for the observer's correction always set to $\tan (\Psi=0)=0$.

Global observability of the proposed state space model can be proven with some simple algebraic calculations. However, it can be shown that the observability of the yaw angle gets lost, if the virtual sensor signal $y_{7}$ is not used in the system's output vector. Thus it is expected that the yaw angle gets estimated with an error, despite the usage of a virtual sensor signal.

For real-time implementation of the observer, the described state space model in continuous time has to be discretized in time. The discretization is performed using the Euler-forward method. The observer itself is realized as a standard EKF. In the following $\mathbf{R}$ and $\mathbf{Q}$ denote the covariance matrices of the sensor noise and the process noise, respectively. Theses matrices are chosen as diagonal matrices and given by

$$
\begin{aligned}
& \mathbf{R}=\operatorname{diag}\left(\begin{array}{lllllll}
r_{\omega} & r_{\omega} & r_{\omega} & r_{a} & r_{a} & r_{a} & r_{\Psi}
\end{array}\right), \\
& \mathbf{Q}=\operatorname{diag}\left(\begin{array}{llllllll}
q_{q} & q_{q} & q_{q} & q_{q} & q_{\omega} & q_{\omega} & q_{\omega} & q_{\rho} \\
q_{\rho} & q_{\rho}
\end{array}\right) .
\end{aligned}
$$

In (13) $r_{\omega}$ and $r_{a}$ denote the sensor noise of each rotation rate sensor and each accelerometer of the IMU, while $r_{\Psi}$ is the sensor noise of the virtual sensor signal for the yaw angle. In (14) $q_{q}, q_{\omega}$, and $q_{\rho}$ are the process noise of the 
four elements of the quaternion, each rotation rate, and each corresponding offset term.

The elements of $\mathbf{R}$ are used in the following to reflect uncertainties of different sensor signals. The elements $r_{\omega}$ and $r_{\Psi}$ are chosen to be constant with $r_{\Psi} \gg r_{\omega}$ assigning more uncertainties to the virtual sensor than to the rotation rate sensors. As already mentioned, the output equations of the accelerometers (7) are only valid during phases when the IMU is only affected by the earth's gravitational vector. On the other hand, this means that the measured accelerations are very uncertain if the IMU is accelerated by other external forces. To take these situations into account, the accelerometers' sensor noise $r_{a}$ is adapted to different situations as proposed by Favre et. al [11]. However, to avoid hard switches between different parameter values as it was done by Favre, $r_{a}$ is adapted linearly to situations when external forces act on the IMU. Such situations are characterized by the difference of the absolute value of the earth's gravitational vector $\tilde{\mathbf{g}}$ and the absolute value of the measured accelerations $\tilde{\mathbf{a}}=\left\|\mathbf{a}_{\text {imu }}^{b}\right\|$ as well as the absolute value of the measured rates $\tilde{\boldsymbol{\omega}}=\left\|\boldsymbol{\omega}_{\text {imu }}^{b}\right\|$, if the IMU is not located in the center of gravity of the ship. Thus, $r_{a}$ is given by

$$
r_{a}=\underline{r}_{a}+\beta_{a} \alpha_{\varepsilon_{a}}(|\tilde{\mathbf{a}}-\tilde{\mathbf{g}}|)+\beta_{\omega} \alpha_{\varepsilon_{\omega}}(\tilde{\boldsymbol{\omega}}),
$$

where $\underline{r}_{a}$ is the minimum value of $r_{a}$ during non-accelerated situations, $\beta_{a}, \beta_{\omega}>0$ are the linear adaption coefficients, and $\alpha_{\varepsilon}(\eta)$ is defined by

$$
\alpha_{\varepsilon}(\eta)= \begin{cases}\eta-\varepsilon, & \text { if } \eta>\varepsilon \\ 0, & \text { if }-\eta \leq \varepsilon \leq \eta \\ \eta+\varepsilon, & \text { if } \eta<-\varepsilon\end{cases}
$$

to generate a dead band where $r_{a}$ is not adapted. The parameters $\beta_{a}$ and $\beta_{\omega}$ are obtained from experiments and chosen in a way that $r_{a} \gg \underline{r}_{a}$ holds during phases when the IMU is accelerated by forces other than the earth's gravitation.

The elements $q_{q}$ and $q_{\rho}$ of the diagonal matrix $\mathbf{Q}$ given in (14) are selected to be constant with $q_{q} \gg q_{\rho}$, as the offset terms are almost constant compared to the quaternion. The process noise of the rotation rates $q_{\omega}$ is equal to the unknown white noise parameters of (3). To guarantee a fast dynamic of the states corresponding to the rotation rates during fast rotations, the process noise $q_{\omega}$ is adapted to the absolute value of the measured rates. It can be expressed as

$$
q_{\omega}=\underline{q}_{\omega}+\kappa_{\omega} \alpha_{\varepsilon_{\omega}}(\tilde{\boldsymbol{\omega}})
$$

with the minimum value $\underline{q}_{\omega}$ and $\alpha_{\varepsilon}(\eta)$ as defined in (16). The linear adaption coefficient $\kappa_{\omega}$ is obtained from experiments.

\section{RESULTS}

In the following the proposed observer for ship's attitude estimation is evaluated. Special attention is given to the performance of estimating the roll and pitch angle, as these angles are required for active heave compensation during subsea lifting operations. First, some simulation results are

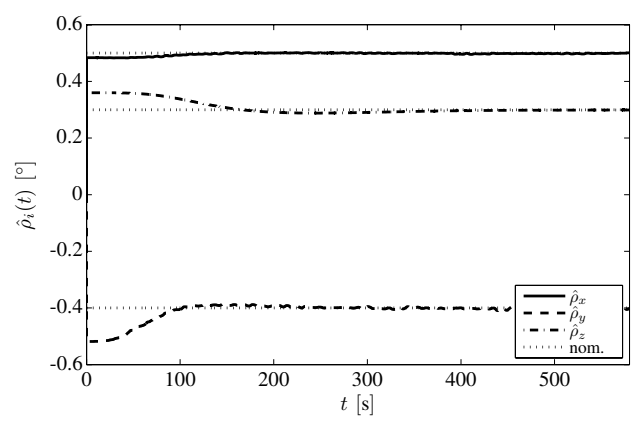

Fig. 2. Estimated offsets $\hat{\rho}_{x}, \hat{\rho}_{y}$, and $\hat{\rho}_{z}$ of the rotation rate sensors together with their nominal values during simulation.

given. After that measurement results from an experimental setup are presented.

\section{A. Simulation}

The motion sequence used for simulation is obtained from a six degrees of freedom simulation of a ship [12]. The model used in the following corresponds to a ship that is $82.8 \mathrm{~m}$ long and has 6,362 tons of weight. The model is excited with a JONSWAP spectrum with a significant wave height of $4 \mathrm{~m}$. The resulting rotation rates in the $b$-frame are disturbed with a constant offset and sensor noise; the translational accelerations are also disturbed with sensor noise.

It is clear that the performance of the attitude estimation depends mainly on the correct offset estimation of the rotation rate sensors. Fig. 2 shows the estimated offsets of all three sensors in the $b$-frame together with the nominal ones used in the simulation. The initial values for the estimated offsets $\hat{\rho_{x}}(0), \hat{\rho_{y}}(0)$, and $\hat{\rho_{z}}(0)$ are obtained from an initialization phase at the beginning. During this phase the rotation rate sensor's mean values are taken over a certain time horizon and used to initialize each state to reduce the observer's decay time. In the following $\hat{\bullet}$ denotes the estimated values of a certain state. As can be seen, the estimated offsets $\hat{\rho}$ converge to the nominal offsets after a short decay time. Furthermore, the estimated offsets are almost constant after they reached their nominal values. This also holds for the offset of the $z$-axis $\hat{\rho}_{z}$, which can be attributed to the virtual sensor for the yaw angle. Since the performance of the offset estimation is good, it is expected that the ship's attitude is also estimated correctly.

Fig. 3 compares the estimated Euler angles $\hat{\Phi}, \hat{\Theta}$, and $\hat{\Psi}$ with the simulated ones for the same simulation as in Fig. 2. For a better depiction, the figure only shows a time slot of the whole sequence after the initial errors are decayed. Figs. 3a and $3 b$ show that the error of the estimated pitch angle $\hat{\Theta}$ is slightly higher than the error of the estimated roll angle $\hat{\Phi}$. That is due to the fact that the absolute pitch motion are bigger than the absolute roll motion. Nevertheless, both errors are in an acceptable range. Fig. 3c indicates that the yaw angle gets also estimated with an tolerable error, however the performance is not as good as the performance for the roll and pitch angles. The maximum errors and the Root Mean Square (RMS) errors for each axis are 


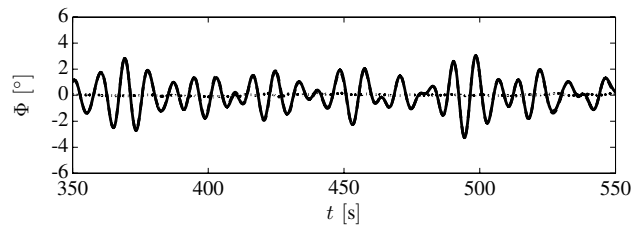

(a) $\Phi$ and $\hat{\Phi}$

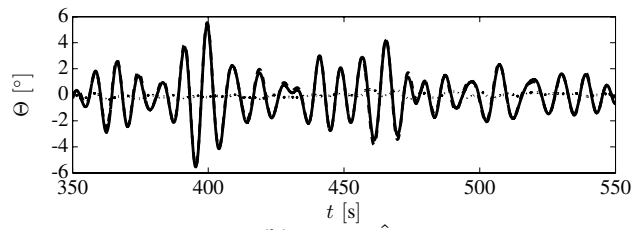

(b) $\Theta$ and $\hat{\Theta}$.

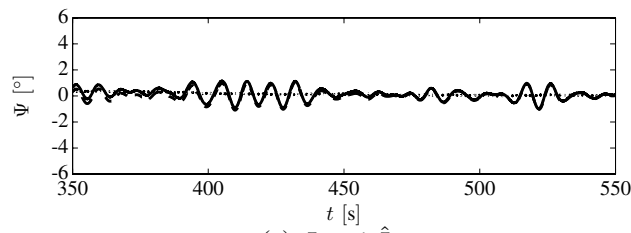

(c) $\Psi$ and $\hat{\Psi}$

Fig. 3. Simulated (dashed line) and estimated (solid line) Euler angles together with their estimation errors (dotted line).

TABLE I

SIMULATED ESTIMATION ERRORS

\begin{tabular}{|c|c|c|c|}
\hline & $\Phi\left[^{\circ}\right]$ & $\Theta\left[^{\circ}\right]$ & $\Psi\left[^{\circ}\right]$ \\
\hline max. error & 0.16 & 0.42 & 0.37 \\
\hline RMS error & 0.06 & 0.16 & 0.18 \\
\hline max. amplitude & 3.03 & 5.55 & 1.04 \\
\hline
\end{tabular}

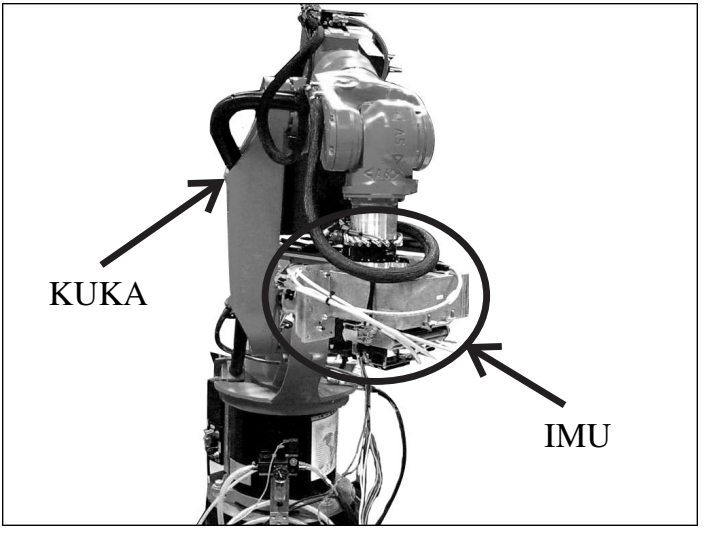

Fig. 4. Experimental setup consisting of a KUKA robot and an IMU from Analog Devices (ADIS 16365).

summarized in Tab. I together with the maximum amplitude of the corresponding motion sequence.

\section{B. Experimental setup}

The experimental setup used to evaluate the algorithm for attitude estimation consists of an IMU from Analog Devices (ADIS 16365) and a KUKA robot. The accelerometers of the IMU have a range of $\pm 18 g$ and the measurement noise is rated at $0.5 \mathrm{mg} / \sqrt{\mathrm{Hz}}$. The gyros have a range of $\pm 150^{\circ} / \mathrm{s}$ and a noise rating of $0.044^{\circ} / \mathrm{s} / \sqrt{\mathrm{Hz}}$ [13]. The reference

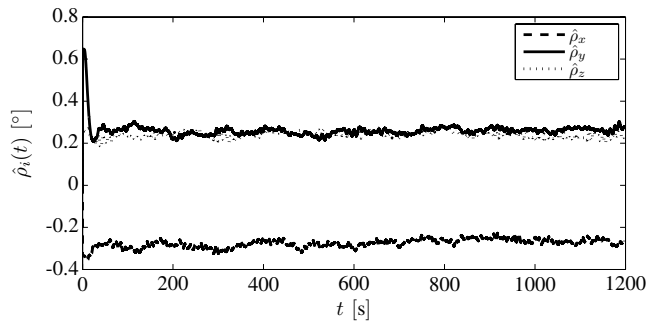

Fig. 5. Estimated offsets $\hat{\rho}_{x}, \hat{\rho}_{y}$, and $\hat{\rho}_{z}$ of the rotation rate sensors obtained from the experimental setup.

TABLE II

ESTIMATION ERRORS FROM EXPERIMENTAL SETUP

\begin{tabular}{|c|c|c|c|}
\hline & $\Phi\left[^{\circ}\right]$ & $\Theta\left[^{\circ}\right]$ & $\Psi\left[^{\circ}\right]$ \\
\hline max. error & 0.39 & 0.29 & 0.44 \\
\hline RMS error & 0.14 & 0.10 & 0.15 \\
\hline max. amplitude & 8.36 & 5.13 & 1.68 \\
\hline
\end{tabular}

paths for all six degrees of freedom of the KUKA robot are generated with the same ship model used for simulation. The simulated motion are recorded and used afterward to move the KUKA robot. The measured IMU signals are used for an online estimation of the IMU's attitude with the proposed method. The estimated Euler angles are compared to the angles obtained from the incremental encoders of the KUKA robot denoted as reference signals in the following. Fig. 4 shows the complete setup.

Fig. 5 presents the estimated offsets $\hat{\rho}$ of all three rotation rate sensors. In contrast to the simulation results, the nominal values of the offsets are unknown. Thus Fig. 5 only shows the estimated values. However, it indicates that after a short decay time every offset oscillates around a constant value, as it is expected from simulation. In addition, the figure clearly illustrates the effect of the virtual sensor for the yaw angle. The offset of the $z$-axis $\hat{\rho}_{z}$ does not diverge away.

Fig. 6 illustrates the corresponding Euler angles for 900s $\leq$ $t \leq 1100$ s. Figs. $6 a$ and $6 \mathrm{~b}$ obviously show that the estimated roll and pitch angles are in good accordance with the reference signals, while Fig. 6c depicts that the yaw angle does not diverge and oscillates around zero. Again, Tab. II summarizes the maximum error values, the RMS errors and the maximum amplitudes of the motion sequences for each axis. From the results follows that the estimation error of the roll and pitch angles stay beyond acceptable values. The error for the yaw axis is relatively higher; however the correct estimation of the yaw angle is not required for active heave compensation systems as mentioned above.

The adapted values for the sensor noise of the accelerometers $r_{a}$ and the process noise of the rotation rates $q_{\omega}$ as given in (15) and (17) are demonstrated in Fig. 7 for the test sequence. The figure shows that $q_{\omega}$ is almost constant, while $r_{a}$ changes due to the absolute value of the measured accelerations $\tilde{\mathbf{a}}$ which is given in Fig. 8 . 


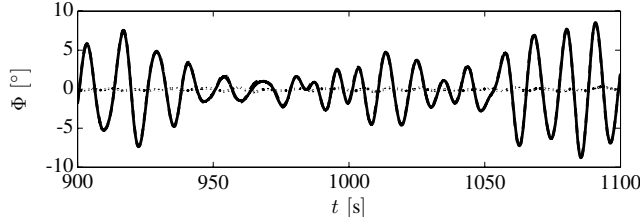

(a) $\Phi$ and $\hat{\Phi}$.

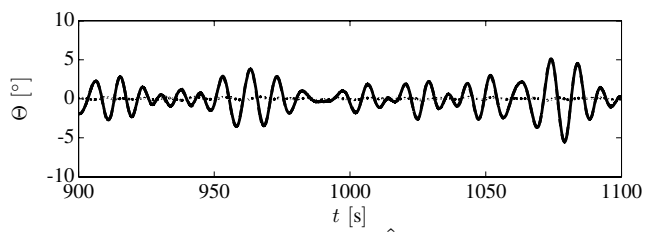

(b) $\Theta$ and $\hat{\Theta}$.

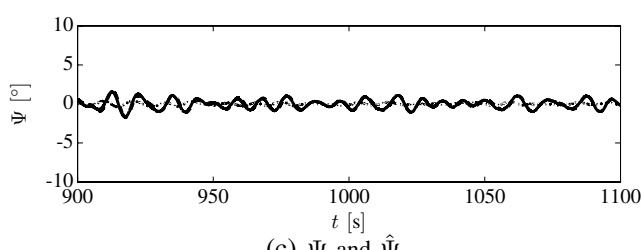

(c) $\Psi$ and $\hat{\Psi}$.

Fig. 6. Estimated Euler angles (solid line) and the corresponding references (dashed line) obtained from the experimental setup. The estimation errors are also depicted (dotted line).

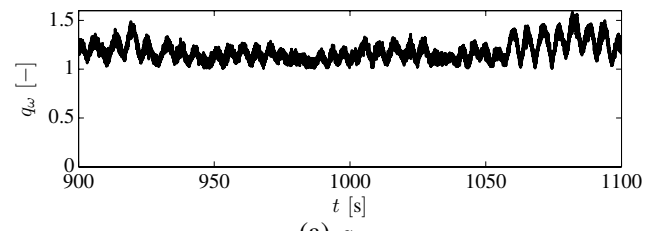

(a) $q_{\omega}$.

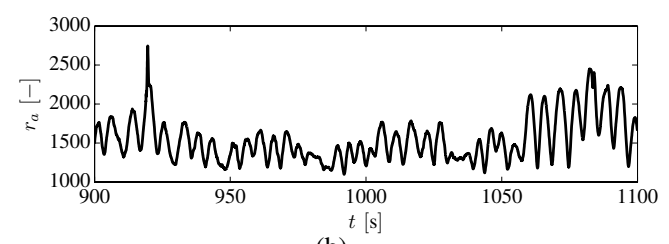

(b) $r_{a}$.

Fig. 7. Adapted sensor noise of the accelerometers $r_{a}$ and adapted process noise of the rotation rates $q_{\omega}$ used for the EKF.

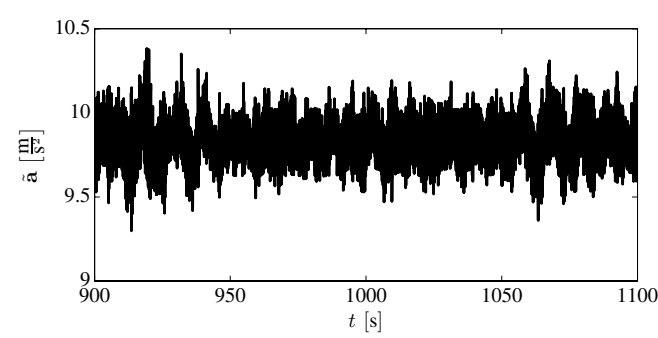

Fig. 8. Absolute value of the measured accelerations ã used to adapt the sensor noise $r_{a}$.

\section{CONCLUSION}

This paper has presented an attitude estimation approach for ships or vessels used during subsea lifting operations. Since active heave compensation systems require actual roll and pitch motion of a vessel, special attention was given to these motion. The derived approach fuses the rotation rates of an IMU with its accelerometer measurements to estimate the vessel's attitude. To stabilize the unobservable yaw motion around zero, a virtual sensor signal for the yaw angle is applied. The parameters of the EKF are adapted online and depend on the measured absolute values of the accelerometers and rotation rate sensors.

Through simulation and measurement results, the attitude estimation algorithm was validated. It was shown that the estimated vessel's roll and pitch motion are in good accordance with the reference signals. Furthermore, the estimation error of the yaw angle was also in an acceptable range.

\section{REFERENCES}

[1] S. Küchler, T. Mahl, J. Neupert, K. Schneider, and O. Sawodny, "Active Control for an Offshore Crane Using Prediction of the Vessel's Motion," IEEE/ASME Transactions on Mechatronics, vol. 16, no. 2, pp. 297-309, 2011.

[2] K. Do and J. Pan, "Nonlinear control of an active heave compensation system," Ocean Engineering, vol. 35, no. 5-6, pp. 558 - 571, 2008.

[3] S. Messineo and A. Serrani, "Offshore crane control based on adaptive external models," Automatica, vol. 45, no. 11, pp. 2546 - 2556, 2009.

[4] J.-M. Godhavn, "Adaptive tuning of heave filter in motion sensor," in Proceedings of OCEANS, Nice, 1998, pp. $174-178$.

[5] — - "High quality heave measurements based on GPS RTK and accelerometer technology," in Proceedings of MTS/IEEE OCEANS, vol. 1, Providence, RI, 2000, pp. $309-314$.

[6] T. Fossen and T. Perez, "Kalman filtering for positioning and heading control of ships and offshore rigs," IEEE Control Systems Magazine, vol. 29, no. 6, pp. $32-46,2009$.

[7] A. Kim and M. Golnaraghi, "A quaternion-based orientation estimation algorithm using an inertial measurement unit," in Proceedings of Position Location and Navigation Symposium, Monterey, CA, 2004, pp. $268-272$.

[8] N. Metni, J.-M. Pflimlin, T. Hamel, and P. Soures, "Attitude and gyro bias estimation for a vtol uav," Control Engineering Practice, vol. 14, no. 12 , pp. 1511 - 1520, 2006.

[9] P. Setoodeh, A. Khayatian, and E. Frajah, "Attitude estimation by separate-bias kalman filter-based data fusion," The Journal of Navigation, vol. 57, no. 2, pp. 261-273, 2004.

[10] D. Titterton and J. Weston, Strapdown Inertial Navigation Technology, 2nd ed., N. Stewart and H. Griffiths, Eds. Stevenage, United Kingdom: Institution of Electrical Engineers, 2004.

[11] J. Favre, B. Jolles, O. Siegrist, and K. Aminian, "Quaternion-based fusion of gyroscopes and accelerometers to improve $3 \mathrm{~d}$ angle measurement," Electronics Letters, vol. 42, no. 11, pp. 612-614, 2006.

[12] MSS, "Marine systems simulator," http://www.marinecontrol.org, 2010, viewed 20.11.2010.

[13] ADIS 16365, Six Degrees of Freedom Inertial Sensor, Analog Devices, 2009. 